\title{
GENETIC ANALYSIS OF SILVER-FIR POPULATIONS IN THE NORTH CARPATHIAN AND SUDETEN MOUNTAINS
}

\author{
LEON MEJNARTOWICZ \\ Polisch Academy of Sciences, Institute of Dendrology \\ Parkowa 5, 62-035 Kórnik, Poland \\ e-mail: idkornik@man.poznan.pl \\ (Received: March 18, 2004. Accepted: May 18, 2004)
}

\begin{abstract}
Seeds collected from individual trees in the 16 Carpathian and 2 Sudeten silver-fir (Abies alba) populations were studied with the starch gel electrophoresis in megagametophytes using 14 enzyme systems with 28 loci. The results show that the geographical distance between populations are in a small part reflected in genetic distances. There are two main groups of populations: Sudeten and Carpathian with a very big genetic distance between them. Other populations consist of a few small groups with low gene flow between them $\left(N_{m}=3.286\right)$. About $80 \%$ of genetic variation is located within populations $\left(F_{S T}=0.223\right)$. Average values for genetic multiplicity and diversity for Carpathian populations are as follows: number of alleles per locus: $N a=2.308$, with effective number of alleles $\mathrm{Ne}=1.552$ and proportion of polymorphic loci $71.21 \%$. The mean number of alleles per locus $(\mathrm{Na})$ varied from 2.107 to 2.607 in population. The mean effective number of alleles per locus $(\mathrm{Ne})$ ranged from 1.429 to 1.662 . Average $F_{i s}$ for Carpathian populations was -0.021 , which means that there is small excess of heterozygotes. The average observed heterozygosity amounted to $H o=0.275$ and expected heterozygosity was $H e=$ 0.269. The dendrogram structure and presence of rare alleles found in silver-fir of Czech, and Slovakian populations allow for a hypothesis that in postglaciation the silver-fir moved into the Polish Carpathians not westward from the east but from the south along river valleys from some Balkan refuges, getting North bypassing the High Tatra Range. This way, a highly diversified set of populations originated, differ in the presence of rare alleles. This differentiation is not prevented by a relatively small flow of genes between populations. The calculated gene flow $N_{m}=3.286$ also indicates isolation between the populations. It means 3.3 immigrants per generation into the studied populations.
\end{abstract}

KEY WORDS: Abies alba, silver-fir isozymes, heterozygosity, genetic diversity, genetic distance, Carpathian Mts., Sudeten Mts.

\section{INTRODUCTION}

The northern and eastern limits of the occurrence of silver-fir (Abies alba Mill.) passes through Poland's territory with the Sudeten and Carpathian Mountains (Fig. 1). The Polish north Carpathian arc is formed by different groups of mountain chains called Western and Eastern Beskids. Many of these mountains are separated by deep valleys formed in the last glaciation. This type of orographic formation of the Beskids results in differentiations among forest populations including the silver-fir populations (Mejnartowicz 2003).

Till the end of the $17^{\text {th }}$ century silver-fir was common in the forests of the Sudeten and Carpathians. Then, predatory exploitation, improper forest management, environment pollution, and some unclear natural factors led to the species decline, known as "silver-fir declining", on the entire area of European forests. Since about 1995 some recovery of the silver-fir has been noticed on the territory of Polish Carpathians.

In the western Sudeten Mts and in a major part of Saxony mountains, silver-fir became extinct and as a result appeared an anthropogenic disjunction in the population range of this species (Mejnartowicz 1983). It was believed that one of the reasons for the decline in the silver-fir stand was the species significant homozygosity (Kantor and Vincent 1970; Larsen 1986; Obmiński 1977), as silver-fir possesses one of the heaviest pollen grains. This should have caused high degree of self-pollination resulting in self-fertilization. This hypothesis was discredited in the early isozymes studies on the genetic structure of the silver-fir population (Mejnartowicz 1979). Populations of the species exhibited great genetic variation and diversity, with values comparable with those described in pine populations, and even higher than in spruce populations (Bergmann et al. 1990; Breitenbach-Dorfer et al. 1997; Konnert et al. Berg- 


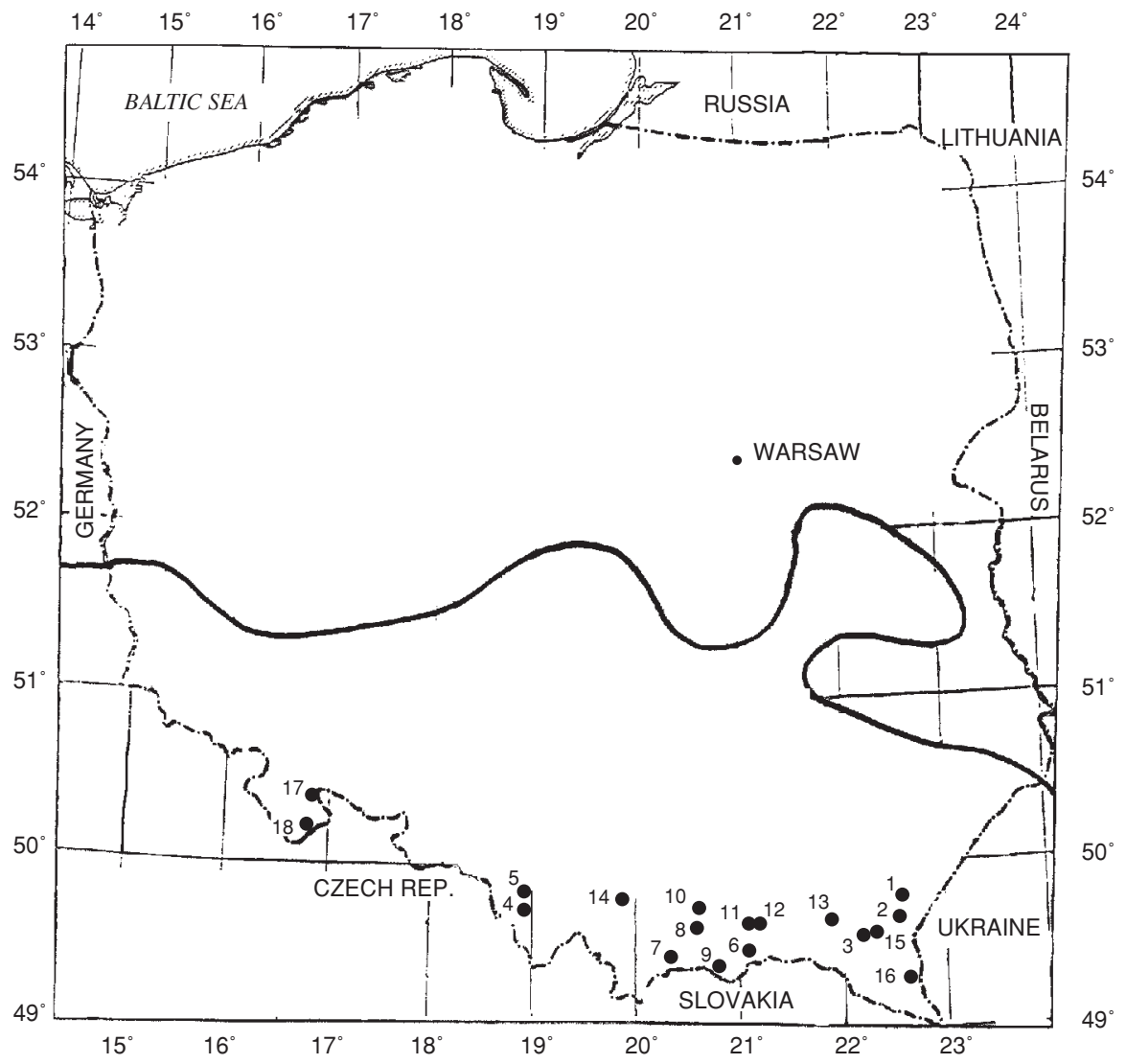

Fig. 1. North-eastern border of silver-fir distribution. Location of the investigated population 1-18. mann 1995; Lewandowski et al. 2001; Liepelt et al. 2002; Mejnartowicz 1979a, 1996, 2000; Schroeder 1989).

\section{MATERIAL AND METHODS}

Seed samples for the analysis came from 16 silver-fir populations from the Eastern and Western Beskids. For the genetic distance comparison data published earlier were included on the two Sudeten populations (Mejnartowicz 2000). Single-tree samples were collected from a minimum 20 trees per population. In all populations the sampled tre- es were from 80 to 160 years old. Geographic location and coordinates of the studied populations as well as age of the trees are shown in Table 1 and Figure 1.

Each tree was genotyped using at least six megagametophytes. Isoenzyme was separated by horizontal starch gel $(11.5 \%)$ electrophoresis in a discontinuous buffers system. For the method literature review, see Mejnartowicz (2000) and Mejnartowicz and Bergmann (2003). In the presented work, allele numbering corresponds to isoenzyme $R f$ : the fastest allozyme migrating toward anode was marked 1 , next -2 , and so on. To estimate genetic variation the following parameters were used: mean number of alleles per lo-

TABLE 1. Geographic coordinate and the tree age of investigated silver-fir populations.

\begin{tabular}{|c|c|c|c|c|c|}
\hline Population name & Geographic region & Latitude & Long. & Altiude [m] & Tree / Age \\
\hline 1. Krasiczyn-Cisowa & Pogórze Przemyskie & $49^{\circ} 43^{\prime}$ & $23^{\circ} 38^{\prime}$ & 360 & 100 \\
\hline 2. Bircza-Wojtkówka & Pogórze Przemyskie & $49^{\circ} 34^{\prime}$ & $22^{\circ} 34^{\prime}$ & $425-500$ & 100 \\
\hline 3. Lesko-Malinki & Góry Słonne & $49^{\circ} 30^{\prime}$ & $22^{\circ} 17^{\prime}$ & $320-340$ & 110 \\
\hline 4. Ustroń-Bukowa 2 & Beskid Śląski & $49^{\circ} 40^{\prime}$ & $18^{\circ} 51^{\prime}$ & 550 & 150 \\
\hline 5. Ustroń-Bukowa 1 & Beskid Śląski & $49^{\circ} 40^{\prime}$ & $18^{\circ} 51^{\prime}$ & 670 & 150 \\
\hline 6. Krynica-Tylicz & Beskid Sądecki & $49^{\circ} 22^{\prime}$ & $20^{\circ} 57^{\prime}$ & 625 & 120 \\
\hline 7. Szczawnica-Krościenko & Beskid Sądecki & $49^{\circ} 25^{\prime}$ & $20^{\circ} 29^{\prime}$ & 610 & 100 \\
\hline 8. Stary Sącz-Przysietnica & Beskid Sądecki & $49^{\circ} 30^{\prime}$ & $20^{\circ} 37^{\prime}$ & 590 & 140 \\
\hline 9. Piwniczna-Łomnica & Beskid Sądecki & $49^{\circ} 27^{\prime}$ & $20^{\circ} 45^{\prime}$ & 580 & 140 \\
\hline 10. Stary Sącz-Kamieniec & Beskid Sądecki & $49^{\circ} 34^{\prime}$ & $20^{\circ} 38^{\prime}$ & 385 & 120 \\
\hline 11. Nawojowa-Berest I & Beskid Niski & $49^{\circ} 33^{\prime}$ & $20^{\circ} 57^{\prime}$ & $625-760$ & 105 \\
\hline 12. Nawojowa-Berest II & Beskid Niski & $49^{\circ} 33^{\prime}$ & $20^{\circ} 57^{\prime}$ & $575-675$ & 110 \\
\hline 13. Rymanów-Szachty & Beskid Niski & $49^{\circ} 26^{\prime}$ & $21^{\circ} 53^{\prime}$ & 500 & 120 \\
\hline 14. Myślenice-Ukleina & Beskid Makowski & $49^{\circ} 49^{\prime}$ & $19^{\circ} 58^{\prime}$ & $260-540$ & 120 \\
\hline 15. Lesko-Czarny Dział & Góry Słonne & $49^{\circ} 30^{\prime}$ & $22^{\circ} 24^{\prime}$ & 500 & 100 \\
\hline 16. Stuposiany-Czereszenka & Bieszczady & $49^{\circ} 09^{\prime}$ & $22^{\circ} 41^{\prime}$ & $640-740$ & 135 \\
\hline 17. Międzylesie & Góry Bystrzyckie & $50^{\circ} 14^{\prime}$ & $16^{\circ} 46^{\prime}$ & 750 & $100-140$ \\
\hline 18. Lądek Zdrój & Góry Złote & $50^{\circ} 21^{\prime}$ & $16^{\circ} 52^{\prime}$ & 700 & $80-160$ \\
\hline
\end{tabular}


cus $(\mathrm{Na})$, effective number of alleles at locus $(\mathrm{Ne})$, number of rare alleles $(\mathrm{NoR} a)$ with the $\mathrm{p}<5 \%$, per cent of polymorphic loci (\%Pol), Shannon's index of genetic diversity $(\mathrm{I})$, observed $(\mathrm{Ho})$ and expected $(\mathrm{He})$ heterozygosity, Wright's fixation index $(F)$, and gene flow $(\mathrm{Nm})$. The statistical analysis of allele frequencies and cluster analysis were performed with the Yeh and Yang (1999) POGENE-32 program.

\section{RESULTS AND DISCUSSION}

The electrophoretic analysis of the 14 enzymes in megagametophytes of European silver-fir from the north Carpathians revealed 85 alleles coded in 28 isozymes loci (Table 2). Allele frequencies of the analyzed loci are given in the Table 3. Only in one locus of the twenty-eight studied MEN2 - no variation was detected, neither in the north Carpathians, Sudeten, nor in the Thuringen populations (Hosius at al. 2000; Lewandowski at al. 2001; Mejnartowicz 2000). Variation in this locus was described by Longauer (1994) in many south and east Carpathians populations. Allele MEN-1 was found exclusively in the Krynica-Tylicz population. In the studied populations we found variation in loci PGI1, PGI2 and SOD1, which were previous considered to be monomorphic. From the twenty-eight studied loci, six were semi-monomorphic: GDH, GOT1, PGM1, SOD1, and PGI1 (Table 2). The last locus, PGI1, was without any variation in all populations from central and southern Germany (Konnert 1994), east Poland (Mejnartowicz 1996) as well as in Sudeten Mts (Lewandowski et al. 2001).

\section{COMPARISON EASTERN AND WESTERN CARPATHIANS POPULATIONS}

From the east Carpathians 5 populations were examined: Krasiczyn, Bircza, Lesko-Malinki, Lesko-Czarny Dział, and Stuposiany. Only in Stuposiany and Krasiczyn populations variations were found in locus Pgm1 (Table 3). In all studied population, silver-fir locus SOD1 was monomorphic, but in the population Bircza-Wojtkówka two alleles exist: common Sod1-2 and private Sod1-1. Allele Sod1-1 distinguished Bircza population from the other populations. In the east Carpathian populations there was smaller allelic multiplicity at the considered loci $(\mathrm{Na}=2.286)$ than in the western Carpathian populations $(\mathrm{Na}=2.318)$ but genetic diversity was greater $(I=0.454)$, and higher than expected heterozygosity ( $\mathrm{Ho}=0.29$ vs. $\mathrm{Ho}=0.27)$ and percentage of polymorphic loci $(72.14 \%)$ than was observed in the western Carpathian populations (70.78) (Table 4). Among the 11 examined western Carpathian silver-fir populations the smallest genetic variation and diversity was found in the population from Szczawnica-Krościenko in the Sacdecki Beskid. It had the smallest number of alleles: $N a=2.107$ and $N e=1.429$, and extremely small number of rare alleles: NoRa $=2(\mathrm{p}<5 \%)$, and the lowest expected heterozygosity $\mathrm{He}=0.238$. These are the lowest values both for the eastern and western Carpathian populations (Table 4). Significant differences in the number of alleles can be seen in the Beskid Sądecki populations - from the lowest values in Szczawnica population to the highest in Krynica-Tylicz populations $(\mathrm{Na}=2.536)$. Populations from Beskid Niski and Beskid Makowski are characterized by the number of alleles per locus $(\mathrm{Na}=2.25$ and $\mathrm{Na}=$ 2.179 respectively) smaller than average for the western

TABLE 2. Enzyme systems assayed in macrogametophytes of individual silver-fir trees.

\begin{tabular}{|c|c|c|c|c|c|}
\hline Enzyme system & Abbre. & E.C. number & Invest. Loci & Allele no. & No. of frequent allele \\
\hline \multirow[t]{2}{*}{ Alcohol dehydrogenase } & $\mathrm{ADH}$ & E.C. 1.1 .1 .1 & ADH1 & 5 & 3 \\
\hline & & & $\mathrm{ADH} 2$ & 3 & 2 \\
\hline \multirow[t]{2}{*}{ Esterase } & EST & E.C. 3.1.1.1 & EST2 & 6 & 3 \\
\hline & & & EST3 & 8 & 5 \\
\hline \multirow[t]{2}{*}{ Fluorescence esterase } & FLE & E.C. 3.1.1.2 & FLE1 & 4 & 2 \\
\hline & & & FLE2 & 5 & 2 \\
\hline Glutamate dehydrogen. & GDH & E.C. 1.4 .1 .2 & GDH1 & 2 & 2 \\
\hline \multirow{4}{*}{ Glutamate-oxaloacetate-transaminase } & GOT (AAT) & E.C. 2.6.1.1 & GOT1 & 3 & 2 \\
\hline & & & GOT2 & 3 & 1 \\
\hline & & & GOT3 & 5 & 3 \\
\hline & & & GOT4 & 5 & 4 \\
\hline \multirow[t]{2}{*}{ Isocitrate dehydrogenase } & IDH & E.C. 1.1 .1 .42 & IDH1 & 4 & 3 \\
\hline & & & $\mathrm{IDH} 2$ & 3 & $1 ; 3$ \\
\hline \multirow[t]{2}{*}{ Leucine aminopeptidase } & LAP & E.C. 3.4 .11 .1 & LAP1 & 4 & 2 \\
\hline & & & LAP2 & 8 & $4 ; 5$ \\
\hline \multirow[t]{3}{*}{ Malate dehydrogenase } & $\mathrm{MDH}$ & E.C. 1.1 .1 .37 & MDH1 & 5 & 2 \\
\hline & & & $\mathrm{MDH} 2$ & 5 & $1 ; 3$ \\
\hline & & & MDH3 & 3 & 1 \\
\hline \multirow[t]{2}{*}{ Menadione reductase } & MEN (DIA) & E.C. 1.6 .4 .3 & MEN1 & 3 & 1 \\
\hline & & & MEN2 & 1 & 1 \\
\hline \multirow[t]{2}{*}{ Phosphoglucomutase } & PGM & E.C. 5.4 .2 .2 & PGM1 & 3 & 2 \\
\hline & & & PGM2 & 2 & 2 \\
\hline \multirow[t]{2}{*}{ Phosphoglucose isomerase } & PGI & E.C. 5.3.1.9 & PGI1 & 2 & 2 \\
\hline & & & PGI2 & 4 & $2 ; 3$ \\
\hline \multirow[t]{2}{*}{ 6-Phosphogluconic dehydrogenase } & 6-PGD & E.C. 1.1.1.44 & 6PGD1 & 4 & $2 ; 3 ; 4$ \\
\hline & & & 6PGD2 & 6 & $2 ; 4$ \\
\hline Shikimate dehydrogen. & SHDH & E.C. 1.1 .1 .25 & SHDH1 & 4 & 1 \\
\hline Superoxide dismutase & SOD & E.C. 1.15 .1 .1 & SOD1 & 2 & 2 \\
\hline
\end{tabular}


TABLE 3. Allele frequencies in the macrogametophytes of 18 Abies alba populations. For population names see Table 1.

Populations

\begin{tabular}{llllllllllllllllllllllll}
\hline Locus & Allele & KC & BW & LM & UB2 & UB1 & KT & Sz & Prz & Pi & SK & NB-1 & NB-2 & RS & MU & LC & ST & LZ & Mg
\end{tabular}

\begin{tabular}{|c|c|c|c|c|c|c|c|c|c|c|c|c|c|c|c|c|c|c|c|}
\hline \multirow[t]{5}{*}{ ADH1 } & 1 & 0 & 0 & 0 & 0.075 & 0 & 0.050 & 0 & 0.125 & 0.050 & 0.125 & 0.050 & 0 & 0 & 0 & 0.075 & 0 & - & - \\
\hline & 2 & 0.025 & 0 & 0 & 0.025 & 0.075 & 0.075 & 0.111 & 0.025 & 0.025 & 0 & 0 & 0.050 & 0.050 & 0 & 0.025 & 0 & - & - \\
\hline & 3 & 0.975 & 1 & 1 & 0.850 & 0.925 & 0.875 & 0.806 & 0.850 & 0.900 & 0.850 & 0.925 & 0.900 & 0.950 & 1 & 0.875 & 0.975 & - & - \\
\hline & 4 & 0 & 0 & 0 & 0.025 & 0 & 0 & 0 & 0 & 0 & 0 & 0 & 0.025 & 0 & 0 & 0 & 0.025 & - & - \\
\hline & 5 & 0 & 0 & 0 & 0.025 & 0 & 0 & 0.083 & 0 & 0.025 & 0.025 & 0.025 & 0.025 & 0 & 0 & 0.025 & 0 & - & - \\
\hline \multirow[t]{3}{*}{ ADH2 } & 1 & 0 & 0 & 0 & 0.025 & 0.150 & 0.075 & 0 & 0.025 & 0 & 0 & 0.025 & 0 & 0.050 & 0.150 & 0.025 & 0.175 & - & - \\
\hline & 2 & 1 & 1 & 0.975 & 0.950 & 0.800 & 0.900 & 0.972 & 0.950 & 1 & 1 & 0.950 & 0.950 & 0.950 & 0.850 & 0.975 & 0.825 & - & - \\
\hline & 3 & 0 & 0 & 0.025 & 0.025 & 0.050 & 0.025 & 0.028 & 0.025 & 0 & 0 & 0.025 & 0.050 & 0 & 0 & 0 & 0 & - & - \\
\hline \multirow{5}{*}{ EST2 } & 2 & 0 & 0 & 0 & 0 & 0.025 & 0 & 0 & 0 & 0 & 0 & 0.050 & 0 & 0 & 0 & 0 & 0 & - & - \\
\hline & 3 & 0.750 & 0.800 & 0.550 & 0.700 & 0.800 & 0.550 & 0.888 & 0.500 & 0.675 & 0.775 & 0.725 & 0.725 & 0 & 0.900 & 0.650 & 0.775 & - & - \\
\hline & 4 & 0.025 & 0.100 & 0.025 & 0.075 & 0.100 & 0.075 & 0 & 0.125 & 0.125 & 0 & 0.050 & 0.050 & 0.100 & 0.050 & 0.025 & 0.050 & - & - \\
\hline & 5 & 0 & 0 & 0 & 0 & 0 & 0 & 0 & 0 & 0 & 0 & 0 & 0 & 0.650 & 0 & 0 & 0 & - & - \\
\hline & 6 & 0 & 0 & 0 & 0 & 0 & 0 & 0.056 & 0 & 0.050 & 0 & 0 & 0.025 & 0 & 0 & 0 & 0 & - & - \\
\hline \multirow[t]{7}{*}{ EST3 } & 1 & 0 & 0 & 0 & 0.025 & 0.050 & 0 & 0 & 0 & 0 & 0 & 0 & 0 & 0 & 0.075 & 0 & 0 & 0 & 0 \\
\hline & 2 & 0.025 & 0.050 & 0 & 0 & 0.025 & 0 & 0 & 0 & 0 & 0.025 & 0.025 & 0 & 0.050 & 0 & 0.025 & 0.050 & 0.029 & 0 \\
\hline & 4 & 0 & 0 & 0 & 0 & 0.025 & 0 & 0 & 0 & 0 & 0 & 0 & 0.475 & 0.525 & 0 & 0 & 0 & 0 & 0 \\
\hline & 5 & 0.475 & 0.500 & 0.250 & 0.625 & 0.575 & 0.500 & 0.611 & 0.500 & 0.775 & 0.500 & 0.325 & 0.300 & 0.250 & 0.650 & 0.475 & 0.425 & 0.883 & 0.925 \\
\hline & 6 & 0.025 & 0.025 & 0.187 & 0.050 & 0.150 & 0.125 & 0.111 & 0.075 & 0.075 & 0.100 & 0.050 & 0.125 & 0.125 & 0.025 & 0.125 & 0.325 & 0.029 & 0 \\
\hline & 7 & 0 & 0.100 & 0.063 & 0 & 0.025 & 0.025 & 0 & 0 & 0.025 & 0.050 & 0 & 0.100 & 0.025 & 0.050 & 0 & 0.025 & 0 & 0 \\
\hline & 8 & 0 & 0 & 0 & 0 & 0 & 0 & 0 & 0 & 0 & 0 & 0 & 0 & 0.025 & 0 & 0 & 0 & 0 & 0 \\
\hline \multirow[t]{4}{*}{ FLE1 } & 1 & 0 & 0 & 0.050 & 0 & 0 & 0.050 & 0 & 0 & 0 & 0 & 0.125 & 0 & 0 & 0 & 0.075 & 0 & 0 & 0 \\
\hline & 2 & 1 & 1 & 0.850 & 0.950 & 0.975 & 0.925 & 1 & 0.975 & 0.850 & 1 & 0.875 & 0.975 & 1 & 1 & 0.925 & 1 & 1 & 1 \\
\hline & 3 & 0 & 0 & 0.025 & 0.050 & 0 & 0 & 0 & 0 & 0.100 & 0 & 0 & 0 & 0 & 0 & 0 & 0 & 0 & 0 \\
\hline & 4 & 0 & 0 & 0.075 & 0 & 0.025 & 0.025 & 0 & 0.025 & 0.050 & 0 & 0 & 0.025 & 0 & 0 & 0 & 0 & 0 & 0 \\
\hline \multirow[t]{5}{*}{ FLE2 } & 1 & 0.175 & 0.100 & 0.300 & 0.275 & 0.075 & 0.075 & 0.056 & 0.300 & 0.150 & 0.125 & 0.150 & 0.150 & 0.175 & 0.050 & 0.175 & 0.175 & - & - \\
\hline & 2 & 0.800 & 0.875 & 0.700 & 0.700 & 0.875 & 0.825 & 0.944 & 0.700 & 0.800 & 0.850 & 0.800 & 0.850 & 0.750 & 0.950 & 0.800 & 0.825 & - & - \\
\hline & 3 & 0.025 & 0.025 & 0 & 0.025 & 0.050 & 0.100 & 0 & 0 & 0 & 0.025 & 0 & 0 & 0.075 & 0 & 0.025 & 0 & - & - \\
\hline & 4 & 0 & 0 & 0 & 0 & 0 & 0 & 0 & 0 & 0 & 0 & 0.050 & 0 & 0 & 0 & 0 & 0 & - & - \\
\hline & 5 & 0 & 0 & 0 & 0 & 0 & 0 & 0 & 0 & 0.050 & 0 & 0 & 0 & 0 & 0 & 0 & 0 & - & - \\
\hline \multirow[t]{2}{*}{ GDH } & 1 & 0 & 0.025 & 0 & 0 & 0 & 0.075 & 0 & 0 & 0.025 & 0 & 0 & 0 & 0 & 0.025 & 0 & 0.025 & 0 & 0 \\
\hline & 2 & 1 & 0.975 & 1 & 1 & 1 & 0.925 & 1 & 1 & 0.975 & 1 & 1 & 1 & 1 & 0.975 & 1 & 0.975 & 1 & 1 \\
\hline & 3 & 0 & 0 & 0 & 0 & 0 & 0 & 0 & 0 & 0 & 0 & 0 & 0 & 0 & 0 & 0 & 0 & 0.059 & 0 \\
\hline GOT2 & 1 & 0.800 & 0.975 & 0.850 & 0.975 & 0.975 & 1 & 0.944 & 0.975 & 1 & 0.950 & 0.925 & 0.975 & 0.950 & 1 & 1 & 0.950 & 0.971 & 0.850 \\
\hline & 2 & 0.150 & 0 & 0 & 0 & 0.025 & 0 & 0 & 0.025 & 0 & 0.050 & 0.025 & 0 & 0.050 & 0 & 0 & 0.025 & 0 & 0.100 \\
\hline & 3 & 0.050 & 0.025 & 0.150 & 0.025 & 0 & 0 & 0.056 & 0 & 0 & & 0.050 & 0.025 & 0 & 0 & 0 & 0.025 & 0.029 & 0.050 \\
\hline GOT3 & 1 & 0.300 & 0.400 & 0.225 & 0.200 & 0.375 & 0.250 & 0.083 & 0.075 & 0.275 & 0.225 & 0.300 & 0.275 & 0.375 & 0.475 & 0.125 & 0.225 & - & - \\
\hline & 2 & 0 & 0.025 & 0 & 0 & 0 & 0 & 0 & 0 & 0.025 & 0 & 0 & 0 & 0 & 0 & 0 & 0.025 & - & - \\
\hline & 3 & 0.425 & 0.350 & 0.550 & 0.675 & 0.375 & 0.400 & 0.834 & 0.725 & 0.500 & 0.425 & 0.375 & 0.400 & 0.400 & 0.375 & 0.525 & 0.325 & - & - \\
\hline & 4 & 0.025 & 0.125 & 0.075 & 0 & 0.075 & 0.075 & 0 & 0.125 & 0.025 & 0.150 & 0.150 & 0.225 & 0.075 & 0.025 & 0.275 & 0.100 & - & - \\
\hline & 5 & 0.250 & 0.100 & 0.150 & 0.125 & 0.175 & 0.275 & 0.083 & 0.075 & 0.175 & 0.200 & 0.175 & 0.100 & 0.150 & 0.125 & 0.075 & 0.325 & - & - \\
\hline GOT4 & 1 & 0.150 & 0.175 & 0.200 & 0.075 & 0.100 & 0.175 & 0.278 & 0.300 & 0.175 & 0.125 & 0.150 & 0.125 & 0.225 & 0.250 & 0.100 & 0.125 & - & - \\
\hline & 2 & 0.050 & 0.025 & 0.150 & 0.050 & 0 & 0 & 0 & 0 & 0.050 & 0 & 0.050 & 0 & 0.025 & 0 & 0.150 & 0.050 & - & - \\
\hline & 3 & 0 & 0 & 0 & 0 & 0 & 0 & 0 & 0 & 0 & 0 & 0 & 0 & 0 & 0 & 0 & 0 & - & - \\
\hline & 4 & 0.800 & 0.800 & 0.650 & 0.875 & 0.850 & 0.825 & 0.722 & 0.700 & 0.750 & 0.875 & 0.800 & 0.875 & 0.750 & 0.750 & 0.750 & 0.800 & - & - \\
\hline & 5 & 0 & 0 & 0 & 0 & 0.050 & 0 & 0 & 0 & 0.025 & 0 & 0 & 0 & 0 & 0 & 0 & 0.025 & - & - \\
\hline IDH1 & 1 & 0 & 0 & 0 & 0 & 0.050 & 0 & 0 & 0 & 0 & 0 & 0 & 0 & 0 & 0.025 & 0 & 0 & 0.029 & 0 \\
\hline & 2 & 0.325 & 0.150 & 0.300 & 0.275 & 0.175 & 0.125 & 0.139 & 0.225 & 0.125 & 0.250 & 0.275 & 0.275 & 0.250 & 0.375 & 0.350 & 0.225 & 0.029 & 0.200 \\
\hline & 3 & 0.500 & 0.700 & 0.450 & 0.450 & 0.425 & 0.625 & 0.722 & 0.500 & 0.450 & 0.450 & 0.475 & 0.350 & 0.375 & 0.525 & 0.400 & 0.550 & 0.677 & 0.525 \\
\hline & 4 & 0.175 & 0.150 & 0.250 & 0.275 & 0.350 & 0.250 & 0.139 & 0.275 & 0.425 & 0.300 & 0.250 & 0.375 & 0.375 & 0.075 & 0.250 & 0.225 & 0.265 & 0.275 \\
\hline IDH2 & 1 & 0.525 & 0.575 & 0.375 & 0.400 & 0.400 & 0.525 & 0.472 & 0.425 & 0.425 & 0.475 & 0.425 & 0.475 & 0.200 & 0.500 & 0.450 & 0.575 & 0.088 & 0.275 \\
\hline & 2 & 0 & 0 & 0 & 0 & 0 & 0 & 0 & 0 & 0 & 0 & 0 & 0 & 0 & 0 & 0 & 0 & 0 & 0.025 \\
\hline & 3 & 0.475 & 0.425 & 0.625 & 0.600 & 0.600 & 0.475 & 0.528 & 0.575 & 0.575 & 0.525 & 0.575 & 0.525 & 0.800 & 0.500 & 0.550 & 0.425 & 0.912 & 0.700 \\
\hline LAP1 & 1 & 0.050 & 0 & 0 & 0 & 0.025 & 0 & 0.056 & 0 & 0.025 & 0 & 0 & 0 & 0 & 0 & 0.025 & 0.075 & 0.088 & 0 \\
\hline & 2 & 0.925 & 0.975 & 0.975 & 0.875 & 0.975 & 0.950 & 0.944 & 0.975 & 0.975 & 0.950 & 1 & 1 & 0.950 & 0.975 & 0.875 & 0.900 & 0.883 & 0.950 \\
\hline & 3 & 0.025 & 0.025 & 0.025 & 0.025 & 0 & 0.050 & 0 & 0.025 & 0 & 0.050 & 0 & 0 & 0.050 & 0.025 & 0.100 & 0.025 & 0.029 & 0.050 \\
\hline & 4 & 0 & 0 & 0 & 0.100 & 0 & 0 & 0 & 0 & 0 & 0 & 0 & 0 & 0 & 0 & 0 & 0 & 0 & \\
\hline LAP2 & 1 & 0 & 0 & 0 & 0 & 0 & 0 & 0.083 & 0 & 0 & 0 & 0.025 & 0 & 0 & 0.025 & 0 & 0.050 & - & - \\
\hline & 2 & 0 & 0 & 0.150 & 0 & 0 & 0.025 & 00 & 0 & 0 & 0 & 0 & 0.025 & 0 & 0 & 0 & 0 & - & - \\
\hline & 3 & 0 & 0 & 0 & 0.025 & 0.075 & 0.150 & 0 & 0 & 0 & 0 & 0 & & 0 & 0 & 0 & 0.050 & - & - \\
\hline & 4 & 0.700 & 0.450 & 0.325 & 0.400 & 0.550 & 0.375 & 0.139 & 0.475 & 0.525 & 0.450 & 0.475 & 0.525 & 0.475 & 0.525 & 0425 & 0.375 & - & - \\
\hline & 5 & 0.300 & 0.500 & 0.400 & 0.550 & 0.350 & 0.400 & 0.639 & 0.500 & 0.425 & 0.500 & 0.500 & 0.450 & 0.500 & 0.375 & 0.475 & 0.500 & - & - \\
\hline
\end{tabular}




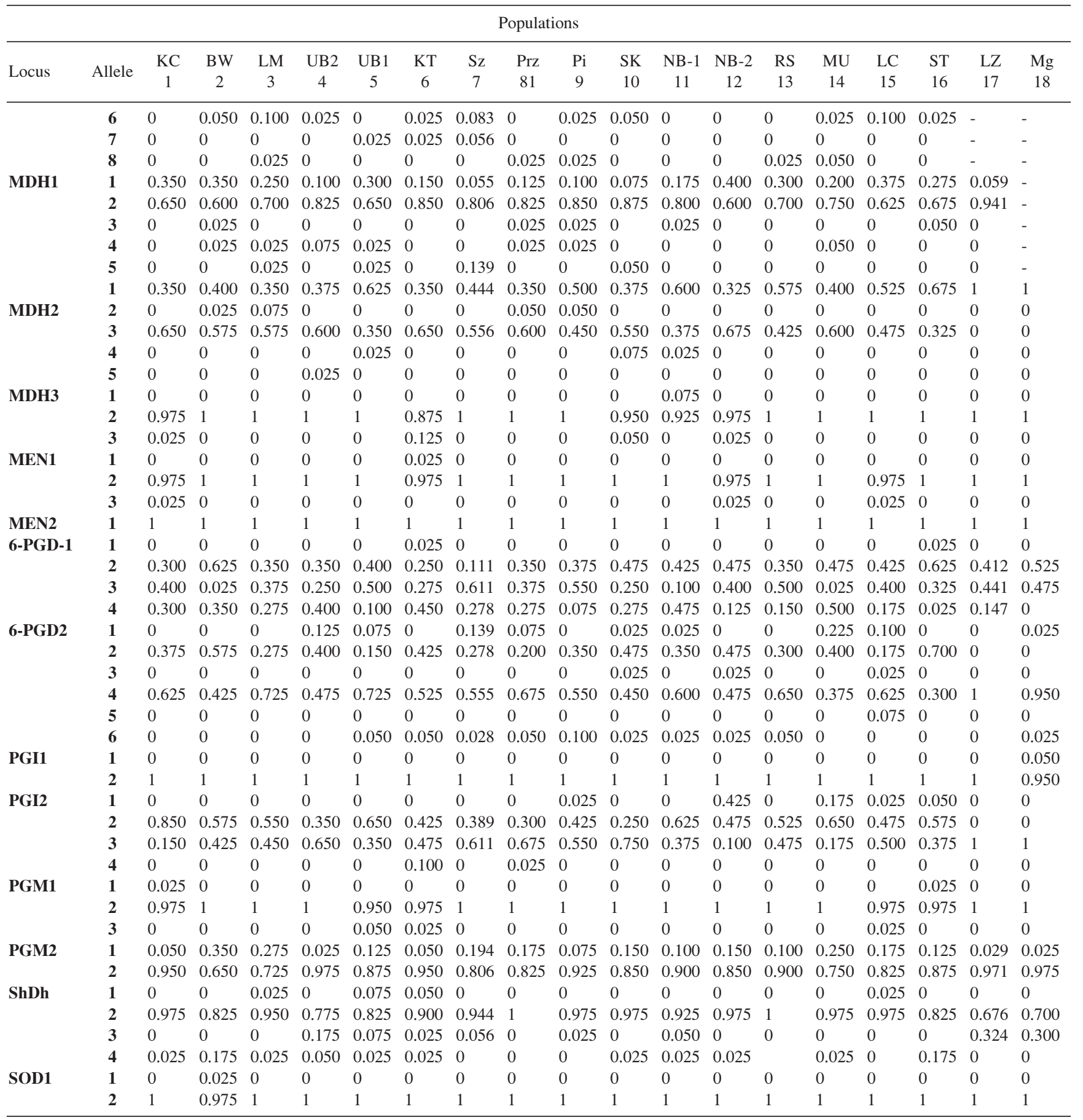

* Population numbers are explained in Table 1.

Carpathians. On average in the Carpathian silver-fir populations we find $N a=2.308$ and $N e=1.552$, which is similar to the data for Germany as presented by Hussendörfer and Konnert (2000).

The Sudeten silver-fir populations have significantly smaller than Carpathian numbers of observed $(\mathrm{Na}=1.731)$ and effective alleles (1.227) (Table 4). Heterozygosity is also higher in the Carpathian populations $(\mathrm{Ho} 0.275, \mathrm{He}=$ 269) than in the Sudeten populations where $H o=130$ and $H e=0.129$ (Table 4). Müller-Starck (1993) and Ziehe and Hattemer (1999) presented results that selection under field conditions is characterized by a viability advantage of those individuals with a large degree of heterozygosity. It was concluded that the value of estimated $(\mathrm{Ho})$ and expected $\mathrm{He}$ ) heterozygosity in a population depends on the age, space, and species composition of the stand (Bergmann 1995; Hussendörfer and Konnert 2000). Evaluation of heterozygosity depends however on the type of analyzed material. Similar heterozygosity values are described for Polish, German, and Italian populations (Bergmann 1995, 1996; Mejnartowicz 1996) when megagametophytes are analyzed. Lower heterozygosity values appear in publications analyzing bud material (Konnert 1994; Lewandowski et al. 2001; Scaltsoyiannes et al. 1999). In the provenance tests the offspring of Szczawnica population is characterized by high survival rate while Lesko-Czarny Dział popu- 
TABLE 4. Summary of Genetic Variation and Heterozygosity for the Polish Carpathians and Sudetes Mts populations of silver-fir.

\begin{tabular}{|c|c|c|c|c|c|c|c|c|}
\hline Population & $N a^{*}$ & $\mathrm{Ne}$ & $I$ & Ho & $\mathrm{He}$ & $F_{i s}$ & $\%$ PoL & $\mathrm{NoRa}$ \\
\hline 1. Krasiczyn-Cisowa & 2.143 & 1.500 & 0.407 & 0.248 & 0.253 & -0.023 & 75.00 & 12 \\
\hline 2. Bircza-Wojtkówka & 2.214 & 1.524 & 0.423 & 0.248 & 0.262 & 0.013 & 67.86 & 11 \\
\hline 15. Lesko-Czarny Dział & 2.357 & 1.605 & 0.479 & 0.366 & 0.291 & -0.163 & 75.00 & 18 \\
\hline 16. Stuposiany-Czereszenka & 2.429 & 1.583 & 0.472 & 0.250 & 0.281 & 0.049 & 75.00 & 18 \\
\hline Mean for East Carpathians & 2.286 & $\mathbf{1 . 5 7 5}$ & 0.454 & 0.29 & 0.277 & -0.039 & 72.14 & 13.8 \\
\hline 4. Ustron-Bukowa 2 & 2.392 & 1.537 & 0.455 & 0.259 & 0.273 & 0.023 & 71.43 & 16 \\
\hline 5. Ustron-Bukowa 1 & 2.607 & 1.562 & 0.484 & 0.234 & 0.276 & 0.080 & 75.00 & 16 \\
\hline Mean for Beskid Śląski & 2.499 & 1.55 & 0.47 & 0.25 & 0.275 & 0.051 & 73.22 & 16 \\
\hline 6. Krynica-Tylicz & 2.536 & 1.625 & 0.501 & 0.295 & 0.295 & -0.018 & 82.14 & 16 \\
\hline 7. Szczawnica-Krościenko & 2.107 & 1.429 & 0.397 & 0.298 & 0.238 & -0.153 & 67.86 & 2 \\
\hline 8. Stary Sącz-Przysietnica & 2.250 & 1.538 & 0.440 & 0.277 & 0.269 & -0.020 & 71.43 & 12 \\
\hline 10. Stary Sącz-Kamieniec & 2.214 & 1.555 & 0.432 & 0.250 & 0.259 & 0.035 & 67.86 & 13 \\
\hline 9. Piwniczna-Łomnica & 2.464 & 1.517 & 0.443 & 0.266 & 0.259 & -0.034 & 67.86 & 13 \\
\hline 11. Nawojowa-Berest 1 & 2.393 & 1.548 & 0.456 & 0.279 & 0.273 & 0.035 & 71.43 & 18 \\
\hline 12. Nawojowa-Berest 2 & 2.250 & 1.588 & 0.443 & 0.261 & 0.270 & -0.005 & 75.00 & 14 \\
\hline 13. Rymanów-Szachty & 2.107 & 1.551 & 0.428 & 0.257 & 0.262 & -0.019 & 64.29 & 10 \\
\hline Mean for Beskid Niski & 2.25 & 1.562 & 0.442 & 0.27 & 0.268 & 0.0037 & 70.24 & 14 \\
\hline 14. Myslenice-Ukleina (Beskid Makowski) & 2.179 & 1.504 & 0.409 & 0.289 & 0.249 & -0.070 & 64.29 & 15 \\
\hline Mean for West Carpathians & 2.318 & 1.541 & 0.444 & 0.270 & 0.266 & -0.013 & 70.781 & 13.2 \\
\hline SD & 0.171 & 0.050 & 0.030 & 0.020 & 0.015 & 0.062 & 5.251 & 4.3 \\
\hline Mean for the Carpathians & 2.308 & 1.552 & 0.448 & 0.275 & 0.269 & -0.021 & 71.21 & 13.5 \\
\hline SD & 0.144 & 0.050 & 0.029 & 0.033 & 0.015 & 0.065 & 4.63 & 3.5 \\
\hline 17. Lądek Zdrój & 1.762 & 1.208 & 0.208 & 0.132 & 0.120 & -0.091 & 35.71 & 7 \\
\hline 18. Międzygórze & 1.700 & 1.246 & 0.228 & 0.127 & 0.138 & 0.010 & 35.71 & 4 \\
\hline Mean for the Sudetes & 1.731 & 1.227 & 0.218 & 0.130 & 0.129 & -0.041 & 35.71 & 5.5 \\
\hline
\end{tabular}

$\mathrm{Na}$ - mean number of observed allele; $\mathrm{Ne}$ - mean number of effective allele; $I$ - shanon's index of genetic diversity; $H o$ - observed heterozygosity; $H e$ - expected heterozygosity; $F_{i s}$ - wright' fixation index; $\% \mathrm{PoL}$ - percent of polymorphic loci; $N o R a$ - number of rare allele.

TABLE 5. Matrix of Nei's genetic distances between the studied Abies alba populations.

\begin{tabular}{|c|c|c|c|c|c|c|c|c|c|c|c|c|c|c|c|c|c|}
\hline pop & ID* & 1 & 3 & 4 & 5 & 6 & 7 & 8 & 9 & 10 & 11 & 12 & 13 & 14 & 15 & 16 & 17 \\
\hline 1 & $* * *$ & & & & & & & & & & & & & & & & \\
\hline 2 & 0.026 & $* * *$ & & & & & & & & & & & & & & & \\
\hline 3 & 0.022 & 0.031 & $* * *$ & & & & & & & & & & & & & & \\
\hline 4 & 0.030 & 0.029 & 0.024 & $* * *$ & & & & & & & & & & & & & \\
\hline 5 & 0.021 & 0.031 & 0.030 & 0.030 & $* * *$ & & & & & & & & & & & & \\
\hline 6 & 0.022 & 0.024 & 0.021 & 0.015 & 0.027 & **** & & & & & & & & & & & \\
\hline 7 & 0.043 & 0.041 & 0.033 & 0.022 & 0.034 & 0.027 & **** & & & & & & & & & & \\
\hline 8 & 0.032 & 0.036 & 0.014 & 0.013 & 0.032 & 0.017 & 0.022 & $* * *$ & & & & & & & & & \\
\hline 9 & 0.028 & 0.033 & 0.027 & 0.016 & 0.014 & 0.019 & 0.023 & 0.017 & **** & & & & & & & & \\
\hline 10 & 0.030 & 0.020 & 0.024 & 0.010 & 0.028 & 0.013 & 0.025 & 0.014 & 0.015 & $* * *$ & & & & & & & \\
\hline 11 & 0.017 & 0.020 & 0.018 & 0.020 & 0.022 & 0.017 & 0.037 & 0.024 & 0.026 & 0.017 & **** & & & & & & \\
\hline 12 & 0.030 & 0.034 & 0.037 & 0.040 & 0.030 & 0.036 & 0.053 & 0.043 & 0.032 & 0.034 & 0.037 & $* * *$ & & & & & \\
\hline 13 & 0.058 & 0.069 & 0.045 & 0.055 & 0.045 & 0.051 & 0.071 & 0.047 & 0.044 & 0.055 & 0.054 & 0.044 & $* * *$ & & & & \\
\hline 14 & 0.023 & 0.015 & 0.038 & 0.030 & 0.028 & 0.027 & 0.042 & 0.042 & 0.034 & 0.028 & 0.022 & 0.032 & 0.073 & **** & & & \\
\hline 15 & 0.021 & 0.027 & 0.013 & 0.018 & 0.017 & 0.021 & 0.027 & 0.013 & 0.018 & 0.016 & 0.016 & 0.028 & 0.044 & 0.032 & *** & & \\
\hline 16 & 0.032 & 0.020 & 0.037 & 0.032 & 0.024 & 0.029 & 0.044 & 0.043 & 0.026 & 0.024 & 0.026 & 0.031 & 0.060 & 0.030 & 0.027 & **** & \\
\hline 17 & 0.246 & 0.240 & 0.205 & 0.182 & 0.176 & 0.194 & 0.193 & 0.173 & 0.168 & 0.192 & 0.202 & 0.252 & 0.184 & 0.242 & 0.187 & 0.212 & $* * *$ \\
\hline 18 & 0.238 & 0.236 & 0.202 & 0.179 & 0.173 & 0.192 & 0.191 & 0.169 & 0.161 & 0.184 & 0.199 & 0.245 & 0.185 & 0.237 & 0.181 & 0.203 & 0.01 \\
\hline
\end{tabular}

* Population numbers are explained in Table 1.

lation has high death rate (Skrzyszewska 2003). It seems that low genetic load of Szczawnica population contributes to its high survival rate, while high genetic load results in high death rate in Lesko-Czarny Dział population. The latter population not only has a great number of rare alleles $(N o R a=18)$ but also high heterozygosity $(H o=0.366)$. It is maintained that rare genes are lethal or sublethal in character and remain in populations mainly in the heterozygotic form. Indeed, all rare alleles detected in northern Carpa- thian and Sudeten silver-fir populations appeared only in the form of heterozygotes. According to Althukov et al. (1987), Scots pine heterozygotes with sublethal genes produce less of the vital seeds than trees with heterozygosity lower than population average. On the other hand though, presence of rare alleles is relevant for the future of a population because they may be playing an adaptative role in the changing environment. The proportion of polymorphic loci $(\% \mathrm{Pol})$, calculated as a measure of genetic variation, 


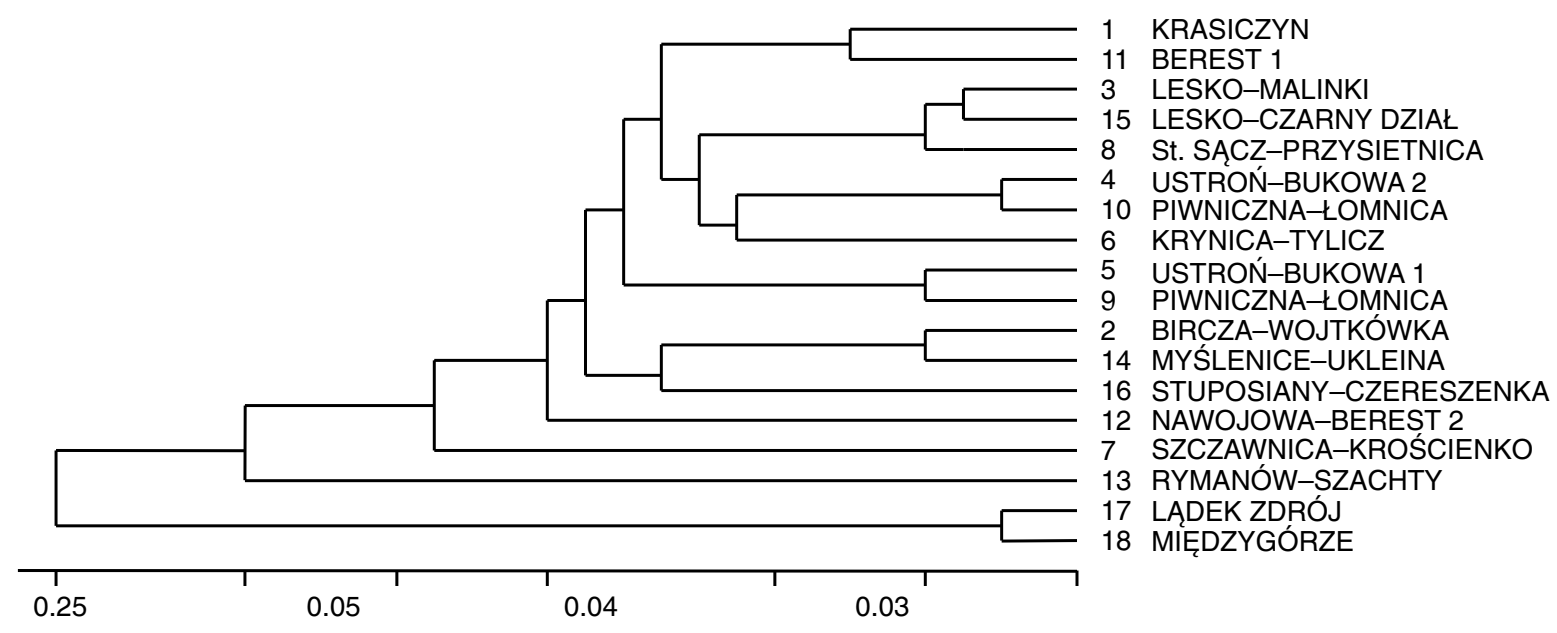

Fig. 2. UPGMA cluster analysis based on genetic distance (Nei 1972) modified from NEIGHBOR procedure of PHYLIP Version 3.5.

for Carpathian populations amounted to $71.21 \%$, which is very similar to the results obtained by Scaltsoyiannes et al. (1999) for Mediterranean firs. The minimum \% Pol $=64.29$ was found in Rymanów and Myślenice populations, the maximum $82.14 \%$ - in Krynica-Tylicz population. For the Sudeten populations \% Pol amounted 35.71.

The $F_{S T}$ coefficient for 27 polymorphic loci ranged from 0.021 at MEN1 to 0.865 at MDH3 with the average $F_{S T}=$ 0.203 , which means that $20 \%$ of the total genetic variation is due to interpopulation diversity and $80 \%$ is located within populations. The inbreeding in the studied silver-fir populations amounted to $2 \%$ only. The heterogeneity of fixation indices suggests no strong influence of inbreeding on the genotypic diversity of all studied silver-fir populations (Table 4).

In the dendrogram drawn according to Nei (1978) on the basis of interpopulation genetic distances (Table 5) two main groups were observed. The first one includes Carpathian silver-fir populations, the other one - Sudeten populations. In the Carpathian group, highly differentiated, the biggest genetic distance in relation to other populations is seen between Rymanów, Szczawnica and Berest-2 populations. The remaining populations form several smaller groups (Fig. 2). The dendrogram structure and presence of rare alleles found in silver-fir of Czech, Moravian and Slovakian populations allow for a hypothesis that in postglaciation the silver-fir moved into the Polish Carpathians not westward from the east but from the south along river valleys from some Balkan refuges, getting north bypassing the High Tatra Range. This way, a highly diversified set of populations originated, differing in the presence of rare alleles. This differentiation is not prevented by a relatively small flow of genes between populations. The calculated gene flow $N_{m}=3.286$ also indicates isolation between the populations. It means 3.3 immigrants per generation into the studied populations.

\section{CONCLUSIONS}

There is a significant diversity within Carpathian silverfir populations. Still greater genetic distance separates $\mathrm{Su}-$ deten and Carpathian populations. Restricted gene flow between populations, their isolation, great differentiation in the silver-fir population environments, and different postglacial immigration routes from the Balkan Peninsula to the Carpathian area result in great interpopulation differences $\left(F_{S T}=0.203\right)$ shown on the dendrogram. Sudeten populations have much lower genetic diversity and great genetic distance compared with Carpathian populations. The results of isoenzyme analyses suggest that Sudeten silver-fir populations derive from populations that in glaciation found refuge on the Apennine Peninsula.

\section{ACKNOWLEDGEMENTS}

This work was financially supported by the project 7/00 of D.G.S.F., Warsaw, and project 6PO4F 35 of CSI and Institute of Dendrology PAS, Kórnik, Poland. The author wish to thank for Prof. Dr. J. Sabor for the part of seed material and J. Kozłowska and M. Ratajczakowa for excellent technical assistance.

\section{LITERATURE CITED}

ALTHUKOV YU.P., GAFAROV N.I., KRUTOVSKII K.V., DUKHAREV V.A. 1987. Allozyme polymorphism in a natural population of Norway spruce (Picea abies Karst.) III. Correlation between levels of individual heterozygosity and relative number of inviable seeds. Sov. Gen. 22: 1580-1585.

BERGMANN F. 1995. Untersuchungen über ökologisch-genetische Anpassungsvorgänge bei der Tanne (Abies alba Mill.) in unterschiedlich immissionbelasteten Regionen unter besonderer Berücksichtigung des Erzgebirges. Teil 2/B. In „Genetik und Waldbau der Weißtanne,.. Schriftenreihe der Sächsischen Landesanstalt. 5: 65-78.

BERGMANN F., GREGORIUS H.-R., LARSEN J.B. 1990. Levels and genetic variation in European silver-fir (Abies alba). Genetica 82: 1-10.

BREITENBACH-DORFER M., KONNERT M., PINSKER W., STARLINGER F., GEBUREK T. 1997. The contact zone between two migration routes of silver-fir, Abies alba (Pinace$a e$ ), revealed by allozyme studies. Pl. Syst. Evol. 206 (1-4): 259-272.

HOSIUS B., BERGMANN F., KONNERT M., HENKEL W. 2000. A concept for seed orchards based on isoenzyme gene markers. Forest Ecology and Management 131 (1-3): 143-152.

HUSSENDÖRFER E., KONNERT M. 2000. Untersuchungen zur genetischen Variation der Weißtanne (Abies alba Mill.) in 
Plenterwäldern im Vergleich zu Altersklassenwäldern. Forstwissenschaftliches Centralblatt 119: 208-225.

KANTOR J., VINCENT G. 1970. Lze předcházet ústupu jedle z našich lesu? Lesnictvi 16 (3): 235-246.

KONNERT M. 1994. Ergebnisse isoenzymatischer Untersuchungen bei der Weißtanne als Entscheidungshilfen für forstliche Maßnahmen. W. Eder (ed). 7. IUFRO Tannen - Symposium 31.10-4.11.1994. WP 1.01-08. Mainz: 30-43.

KONNERT M., BERGMANN F. 1995. The geographical distribution of genetic variation of Silver fir (Abies alba, Pinaceae) in relation to its migration history. Pl. Syst. Evol. 196: 19-30.

LARSEN J.B. 1986. Das Tannensterben: Eine neue Hypothese zur Klärung des Hintergrundes dieser rätselhafte Komplexkrankheit der Weißtanne (Abies alba Mill.) Forstwissenschaftliches Centralblatt 105: 381-396.

LONGAUER R. 1994. Genetic differentiation and diversity of European silver-fir in Eastern part of its natural range. W. Eder (ed.). 7. IUFRO Tannensymposium 31.10-4.11.1994. WP 1.01-08. Mainz: 155-164.

LIEPELT S., BIALOZYT R., ZIEGENHAGEN B. 2002. Winddispersed pollen mediates postglacial gene flow among refugia. Proceedings of the National Academy of Sciences of the United States of America 99 (22): 14590-14594.

LEWANDOWSKI A., FILIPIAK I., BURCZYK J. 2001. Genetic variation of Abies alba Mill. in Polish part of Sudety Mts. Acta Soc. Bot. Pol., 70 (3): 215-219

MEJNARTOWICZ L. 1979. Polymorphism at the LAP and GOT loci in Abies alba Mill. populations. Bulletin de l'Académie Polonaise des Sciences. Série des sciences biologiques. Cl. V. Vol. 27 (12): 1063-1070.

MEJNARTOWICZ L. 1979a. Genetic variation in some isoenzyme loci in Scots pine (Pinus silvestris L.) populations. Arbor. Kórnickie 24: 91-104.

MEJNARTOWICZ L. 1983. Genetyka. Monografia: Jodła pospolita (Abies alba Mill.). Nasze Drzewa Leśne. Ed. S. Białobok. PWN Warszawa-Poznań t. 4: 285-316.

MEJNARTOWICZ L. 1996. Cisovka - the relic population of Abies $a l b a$ and its relationship to man-made silver-fir stands in Białowieża Primeval Forest. Acta Soc. Bot. Pol. 65 (3-4): 319-328.
MEJNARTOWICZ L. 2000. Polish Sudeten and Carpathian Mountains silver-fir (Abies alba) population genetic investigation. IUFRO WP: 1.05-16 Ecology and Silviculture of European Silver Fir. Proc. of the $9^{\text {th }}$ International European Silver Fir Symposium. May 21-26, 2000 Skopje, Rep. of Macedonia, 49$-54$.

MEJNARTOWICZ L. 2003. Genetic analysis of silver-fir populations in the Beskides. Acta Soc. Bot. Pol. 72 (2): 115-119.

MEJNARTOWICZ L., BERGMANN F. 2003. Mode of inheritance of Aspartate aminotransferase in Silver fir (Abies alba Mill.) Silvae Genetica 52 (11): 15-18.

MÜLLER-STARCK G. 1993. Auswirkung von Umweltbelastung auf genetische Strukturen von Waldbestand am Beispiel der Buche (Fagus sylvatica L.). Schriften aus der Forstlichen Fakultät der Uni. Göttingen und der Niedersachsische Forstliche Versuchsanstalt. Bd. 12. 163 pp.

NEI M. 1978. Estimation of average heterozygosity and genetic distance from a small numbers of individuals. Genetics 89: 583-590.

OBMIŃSKI Z. 1977. Ekologia lasu. PWN, Warszawa.

SCHRÖDER S. 1989. Isozyme polymorphisms in silver-fir (Abies alba Mill.). Silvae Genetica 38 (3-4): 130-133.

SCALTSOYIANNES A., TSAKTSIRA M., DROUZAS A.D. 1999. Allozyme differentiation in the Mediterranean firs (Abies, Pinaceae). A first comparative study with phylogenetic implications. Plant Systematics and Evolution 216 (3-4): 289$-307$.

SKRZYSZEWSKA K. 2003. Ocena zmienności przeżywalności jodły w wieku 17 lat na uprawach doświadczenia Jd PL 86/90 In: Ann. Rep Grant No 7/00. „Genetyczna analiza zmienności między i wewnątrzpopulacyjnej jodły pospolitej (Abies alba) w świetle potrzeb hodowli i zachowania zasobów genowych gatunków drzew leśnych" Kraków 2003: 64-75.

YEH F.C., YANG R. 1999. Popgene version 1.31. Freeware for population genetic analysis.

ZIEHE M., HATTEMER H.-H. 1999. Significance of heterozygosity in tree breeding and gene conservation. Forest Tree Improvement 26: 3-25. 